\title{
Microsurgical endoscopy-assisted presigmoid retrolabyrinthine approach as a minimally invasive surgical option for the treatment of medium to large vestibular schwannoma
}

\author{
Domenico d'Avella • Antonio Mazzoni • \\ Elisabetta Zanoletti • Alessandro Martini
}

Received: 9 December 2012 / Accepted: 13 December 2012 / Published online: 13 January 2013

(C) Springer-Verlag Wien 2013

The paper from Iacoangeli and his colleagues from Ancona and Rome in this issue of Acta Neurochirurgica is timely given the continuing controversy on best treatment options for vestibular schwannomas.

Their report is on the preliminary experience with an old approach integrated with the endoscope. The case material includes ten cases of small and medium sized sporadic acoustic neuromas (five cases of 8 and $10 \mathrm{~mm}$, five cases of 11-25 mm), selected on the basis of a favourable anatomy of the temporal bone.

It is noteworthy, and rather exceptional in comparison to other's experiences, that only three cases out of ten had a preoperative normal facial nerve function, while four cases of 11-25 mm had a House-Brackmann (HB) grade 3 and three cases of 10 and $20 \mathrm{~mm}$ had a HB grade 2, which makes $70 \%$ of preoperative facial dysfunction in small and medium-size tumors.

The surgical outcome was outstanding, with unchanged postoperative facial function (with the exception of the case with preoperative radiotherapy), only one case experiencing a hearing loss from AAORLHNS class B to C, and no instances of deafness. The removal of tumors from the internal auditory canal without a drill meatotomy in this series might have been favoured by a limited extension into the canal. The results on the hearing and facial nerve are superior to the results reported in the literature.

We dare to say that we never had a group of ten consecutive cases with only one postop limited hearing loss in over

D. d'Avella $(\bowtie) \cdot$ A. Mazzoni

Neurosurgery, Department of Neurosciences,

University of Padova, Padova, Italy

e-mail: domenico.davella@unipd.it

A. Mazzoni $\cdot$ E. Zanoletti $\cdot$ A. Martini

Otolaryngology, Department of Neurosciences,

University of Padova, Padova, Italy
300 cases of hearing preservation surgery on smaller tumors [8].

This revived approach, which was already dismissed by experienced surgeons, although now implemented by endoscopic assistance needs further validation in order to assess its performance versus the standard retrosigmoid approach.

Notwithstanding, Iacoangeli and his colleagues have further contributed to the debate upon the treatment of acoustic neuroma. This has indeed been an active dispute over the last decades, and is nowadays even keener thanks to the further inputs brought by neurosurgeons, otosurgeons, endoscopists, and, last but not least, radiotherapists.

It should be considered that early diagnosis results in a patient's population mainly (in $70 \%$ of cases, according to accredited studies) made up of small tumors: the current debate and renewal of treatment options is therefore nowadays addressed to such lesions.

The definition of 'small tumor' varies between different Authors: some consider a diameter less than $2 \mathrm{~cm}$ at the cerebello-pontine angle, others consider a diameter of $1,5 \mathrm{~cm}$.

The current issues of the acoustic neuroma treatment debate seem to be:

1- If the goal of treatment is to arrest the disease with no further morbidity and preservation of neurological functions, should this be achieved by radical resection, or the arrest of progression of disease and its functional damage might suffice?

2- Is the collaboration or, better, the cultural and practical integration between Neurosurgeons and Otologists in planning and carrying out surgery a good thing?

3- What role should endoscopic surgery play in the cerebellopontine angle and in the internal auditory canal?

4- In more general terms, how can an early diagnosis actually result in a better prognosis? 
The answer to these questions entails the involvement of several sources by different qualified professionals.

1- The goal of current therapy is recovery with no further morbidity. Clinical observation through periodical MRIs is the treatment option that better exploits the spontaneous growth arrest of the majority of small neuromas [10]. Such attitude can be correct, but bears some important limitations: if hearing is yet not impaired, Hearing Preservation Surgery (HPS) has great chances of success [8], whilst clinical observation determines a progressive loss of hearing [11] even with no tumor growth [5], and reduces the possibilities of late HPS.

Furthermore, if the tumor is larger than $10 \mathrm{~mm}$ at the cerebellopontine angle, surgical removal with preservation of the integrity of the 7th cranial nerve has some limitations, represented by a risk of $17-20 \%$ of HB grade III palsy or worse [1], for lesions between 1.5 and $20 \mathrm{~mm}$.

What is the role of radiosurgery, intended as stereotactic radiosurgery or fractionated stereotactic radiosurgery? It is now indisputable that radiotherapy should be done only if there is evidence of tumor growth, which entails yearly clinical observation with MRI until growth is detectable. Prompt radiotherapy at first diagnosis leads to an inappropriate administration of unneeded radiations, with the potential development of late complications, yet rare, and not well known in the long term. What is the role of radiotherapy, then? Radiotherapy's therapeutic strategy consists in one or more radiotherapic sessions, with further surgery or a second course of radiotherapy if growth recurs. The success of radiotherapy, meant as arrest of tumor growth or of disease progression, is controversial. Indeed, universal data from clinical studies refer to all neuroma types, even to those that do not grow spontaneously. The reported success rate is around $95 \%[6,9]$. This data must be cleansed of the cases of stable tumor. If we consider the current data [10], on the basis of which $2 / 3$ of small tumors doesn't grow, the $5 \%$ rate of therapeutic failure becomes higher than $15 \%$ [7]. We recognize that such figures regarding the "actual" failure rate are arbitrary, arising from the combination of data from different research studies, and serve only to highlight how failures of radiotherapy are somehow higher than expected from the literature, and are still waiting to be proven by clinical expertise. What is the destiny of the failures of radiotherapy, that is the 'rescue surgery' contemplated in radiotherapy's therapeutic plans? Several studies [2-4] agree that surgical removal is more difficult because of the scar tissue and the fragility of the 7th cranial nerve, with significant rates of 7th nerve palsy, incomplete removal and other complications. It must be taken into account that the preservative nature of radiotherapy and clinical observation bears some limitations that we need to be aware of. Surgery following prolonged observation (that sometimes allows tumor sizes which jeopardize an acceptable preservation of 7th nerve), as well as surgery that follows radiotherapy, carry so much morbidity that it contradicts the preservative nature of both. Hearing preservation is nowadays the aim of every therapeutic option, as part of the general goal of not to increase the natural morbidity of the disease. Clinical observation causes a progressive and unpredictable hypoacusis, interesting, in that it is in about half of cases at 5 years follow-up. Radiotherapy as well carries additional hypoacusis, whatever the tumor behaviour. The radiotherapy cases follow up is not able to detect late-onset hypoacusis, because hearing loss is frequent and becomes clinically evident only after the third year, and because the examined frequencies are often less impaired.

In our experience, HPS has the best results only in small tumors (less than $10 \mathrm{~mm}$ in the cerebellopontine angle) and in the cases with preserved hearing, that means equal or better than $30 \mathrm{~dB}$ and 70 of SDS (Speech Discrimination Score), and preserved ABR [8].

2- Collaboration between neurosurgeons and otologists means in fact sharing education, experience, different techniques as well as instrumentation and patient care. Our experience, likewise with others, is widely positive and we recommend as very important that such specialties continue their cooperation in this field.

3- Endoscopic skull base surgery has indisputably already achieved important results, and deserves our attention with regards to its experience in the cerebellopontine angle. The current reports in acoustic neuroma are still preliminary and yet unconvincing. In our opinion, the endoscope in acoustic neuroma microsurgery plays a

Table 1 Algorithm of treatment in acoustic neuroma

\begin{tabular}{lll}
\hline Size & Hearing & Treatment \\
\hline$\leq 10 \mathrm{~mm}$ & $\begin{array}{l}\text { Good hearing } \\
\text { Bad hearing }\end{array}$ & $\rightarrow$ HPS \\
10 to $15 \mathrm{~mm}$ & $\begin{array}{l}\text { (Good/Bad hearing) } \\
\text { MRI 1 year }\end{array}$ & $\begin{array}{l}\text { Growth } \rightarrow \text { Surgery }^{\mathrm{a}} \\
\text { No } \text { growth } \rightarrow \text { Observation }\end{array}$ \\
15 to $25 \mathrm{~mm}$ & & $\rightarrow$ Surgery or RT \\
$>25 \mathrm{~mm}$ & & $\rightarrow$ Surgery \\
\hline
\end{tabular}

Non-cystic acoustic neuroma, size as largest diameter in cerebellopontine angle. RT, radiotherapy, HPS, hearing preservation surgery

${ }^{a}$ Observation involves MRI follow up after 1,2,3,4,5,7,9,14,20 years. Age, comorbidity, surgical risk can be addressed by non-surgical treatment 
collateral role, useful but not essential. The safety and sharpness of microsurgery are out of the endoscope's reach, which pays the penalty for dissection instruments and techniques inferior to the quality of its pictures.

4- Early diagnosis of small neuromas brings us closer to the desirable lack of further morbidity, intended as preservation of hearing and of the 7th cranial nerve. The guidelines that we suggest in Table 1 are the expression of such aim.

Finally, the rehabilitation of lost hearing deserves nowadays some particular considerations. Cochlear implants, when the cochlear nerve is preserved, is an option which from schwannomas in NF2 patients is being extended to sporadic neuromas as well. Auditory brainstem implants shows better results than in the past because of the increasing experience of surgeons and technological progresses of the prosteses. Present therapeutic strategies are already influenced by these restorative surgical modalities.

Conflicts of interest None.

\section{References}

1. Brackmann DE, Cullen RD, Fisher LM (2007) Facial nerve function after translabyrinthine vestibular schwannoma surgery. Otolaryngol Head Neck Surg 136:773-777
2. Friedmann RA, Brackmann DE, Hitselberger WE, Schwartz MS, Iqbal Z, Berliner KI (2005) Surgical salvage after failed irradiation for vestibular schwannoma. Laryngoscope 115:1827-1832

3. Friedman AR, Berliner KI, Bassim M, Ursick J, Slattery WH 3rd, Schwartz MS, Brackmann DE (2011) A paradigm shift in salvage surgery for radiated vestubular schwannoma. Otol Neurotol 32:1322-1328

4. Gerganov VM, Giordano M, Samii A, Samii M (2012) Surgical treatment of patients with vestibular schwannomas after failed radiosurgery. J Neurosurg 116:713-720

5. Graamans K, Van Dijk JE, Jansen LW (2003) Hearing deterioration in patients with a non-growing schwannoma. Acta Otolaryngol 123:51-54

6. Hasegawa T, Fujitani S, Katsumata S, Kida Y, Yoshimoto M, Koike J (2005) Stereotactic radiosurgery for vestibular schwannoma: analysis of 317 patients followed more than 5 years. Neurosurgery 57:257-265

7. Kapoor S, Batra S, Carson K, Shuck J, Kharkar S, Gandhi R, Jackson J, Wemmer J, Terezakis S, Shokek O, Kleinberg L, Rigamonti D (2011) Long-term outcomes of vestibular schwannomas treated with fractionated stereotactic radiotherapy: an institutional experience. Int J Radiat Oncol Biol Phys 81(3):647-653

8. Mazzoni A, Biroli F, Signorelli A, Sortino C, Zanoletti E (2011) Hearing preservation surgery in acoustic neuroma. Slow progress and new strategies. Acta Otorhinolaryngol Ital 31:76-84

9. Regis J, Delsanti C, Roche PH, Thomassin JM, Pellet W (2004) Functional outcomesof radiosurgical treatment of vestibular schwannomas: 1000 successive cases and review of the literature. Neurochirurgie 50:301-311

10. Stangerup SE, Caye-Thomasen P, Tos M, Thomsen J (2006) The natural history of vestibular schwannoma. Otol Neurotol 47:547-552

11. Stangerup SE, Caye-Thomasen P, Tos M, Thomsen J (2008) Change in hearing during "wait and scan" management of patients with vestibular schwannoma. J Laryngol Otol 122:673-681 